\title{
Goal-oriented a posteriori error estimation for conforming and nonconforming approximations with inexact solvers
}

\author{
Gouranga Mallik ${ }^{\dagger}$, Martin Vohralík ${ }^{\star}$, Soleiman Yousef ${ }^{\S}$ \\ $\dagger$ Department of Mathematics \\ Indian Institute of Science Bangalore \\ India 560012 \\ e-mail: gouranga2011@gmail.com \\ web page: https://scholar.google.co.in/citations?user=Wj9vFzMAAAAJ\&hl=en \\ $\star$ Inria \\ 2 rue Simone Iff \\ 75589 Paris, France \\ * CERMICS, Ecole des Ponts \\ 77455 Marne-la-Vallée, France \\ e-mail: martin.vohralik@inria.fr \\ web page: https://who.rocq.inria.fr/Martin.Vohralik/ \\ $\S$ IFP Energies nouvelles \\ 1 \& 4 av. Bois Préau \\ 92852 Rueil-Malmaison, France \\ e-mail: soleiman.yousef@ifpen.fr \\ web page: https://www.ifpenergiesnouvelles.fr/page/soleiman-yousef
}

\begin{abstract}
We derive a unified framework for goal-oriented a posteriori estimation covering in particular higher-order conforming, nonconforming, and discontinuous Galerkin finite element methods, as well as the finite volume method. The considered problem is a model linear second-order elliptic equation with inhomogeneous Dirichlet and Neumann boundary conditions and the quantity of interest is given by an arbitrary functional composed of a volumetric weighted mean value (source) term and a surface weighted mean (Dirichlet boundary) flux term. We specifically do not request the primal and dual discrete problems to be resolved exactly, allowing for inexact solves. Our estimates are based on $\boldsymbol{H}$ (div)-conforming flux reconstructions and $H^{1}$-conforming potential reconstructions and provide a guaranteed upper bound on the goal error. The overall estimator is split into components corresponding to the primal and dual discretization and algebraic errors, which are then used to prescribe efficient stopping criteria for the employed iterative algebraic solvers. Numerical experiments are performed for the finite volume method applied to the Darcy porous media flow problem in two and three space dimensions. They show excellent effectivity indices even in presence of primal and dual algebraic errors and enable to spare a large percentage of unnecessary algebraic iterations.
\end{abstract}

\section{REFERENCES}

[1] Mallik, G., Vohralík, M. and Yousef, S. Goal-oriented a posteriori error estimation for conforming and nonconforming approximations with inexact solvers. J. Comput. Appl. Math., Vol. 366, paper 112367, (2020). 